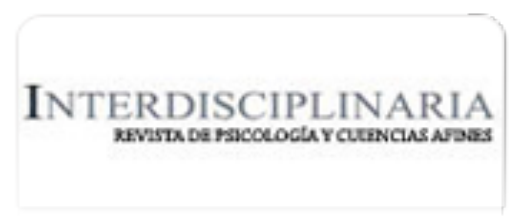

Interdisciplinaria

ISSN: 0325-8203

ISSN: 1668-7027

interdisciplinaria@fibercorp.com.ar

Centro Interamericano de Investigaciones Psicológicas y

Ciencias Afines

Argentina

Robalino Guerra, Paulina E.; Musso, Mariel F.

Inatención del conductor: un estudio acerca de las relaciones entre redes atencionales y la propensión a cometer errores durante la conducción

Interdisciplinaria, vol. 35, núm. 2, 2018, Julio-, pp. 425-444

Centro Interamericano de Investigaciones Psicológicas y Ciencias Afines

Argentina

Disponible en: https://www.redalyc.org/articulo.oa?id=18058785012

Cómo citar el artículo

Número completo

Más información del artículo

Página de la revista en redalyc.org

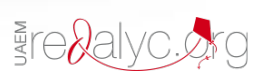

Sistema de Información Científica Redalyc Red de Revistas Científicas de América Latina y el Caribe, España y Portugal Proyecto académico sin fines de lucro, desarrollado bajo la iniciativa de acceso abierto 


\title{
Inatención del conductor: un estudio acerca de las relaciones entre redes atencionales y la propensión a cometer errores durante la conducción*
}

\section{Driver inattention: relationships between attentional networks and propensity to commit errors while driving}

\author{
Paulina E. Robalino Guerra** y Mariel F. Musso***
}

\begin{abstract}
*Las autoras agradecen a la Dra. Silvana Montes de la Universidad Nacional de Mar del Plata - Consejo Nacional de Investigaciones Científicas y Técnicas (CONICET) por aportar el instrumento ARDES y por su asesoramiento, y al Dr. Juan Lupiáñez del Departamento de Psicología Experimental de la Facultad de Psicología de la Universidad de Granada (España) por su predisposición para responder inquietudes.

**Licenciada en Psicología. Becaria Doctoral del Consejo Nacional de Investigaciones Científicas y Técnicas (CONICET) en la Universidad Argentina de la Empresa (UADE). Doctoranda de la Pontífice Universidad Católica Argentina (UCA). Docente Investigador de la Universidad Argentina de la Empresa (UADE).

E-mail: probalino@uade.edu.ar

***Doctora en Psicología. Doctora en Ciencias de la Educación. Investigadora Asistente del Consejo Nacional de Investigaciones Científicas y Técnicas (CONICET) en el Centro Interdisciplinario de Investigaciones en Psicología Matemática y Experimental (CIIPME). Docente Investigador de la Universidad Argentina de la Empresa.
\end{abstract}

Centro Interdisciplinario de Investigaciones en Psicología Matemática y Experimental (CIIPME - CONICET) y Universidad Argentina de la Empresa (UADE). Ciudad Autónoma de Buenos Aires, Argentina.

\section{Resumen}

Los accidentes de tránsito son un fenómeno complejo, resultado de factores ambientales, vehiculares y humanos, y una de las principales causas de muerte a nivel mundial. La inatención es un factor primordial que contribuye a los accidentes de tránsito. El objetivo del presente trabajo fue analizar la relación entre la atención según el modelo de redes atencionales de Posner (1994) y la propensión a cometer errores relacionados con la inatención durante la conducción vehicular. La muestra estuvo compuesta por 70 participantes, edades entre 19 y 59 años, ambos géneros, 9.83 años de experticia como promedio. Se utilizó el Cuestionario de Experiencias durante la conducción (ARDES-ERIC), Test de Redes Atencionales (ANT) y un cuestionario sociodemográfico. Los resultados indi- can que existe una correlación significativa entre el tiempo de reacción (TR) total y la propensión a cometer errores durante la conducción. La interacción entre la experticia y el TR total sobre la propensión a cometer errores fue significativa. La atención ejecutiva tuvo un efecto significativo sobre la propensión a cometer errores y la dimensión de control. El modelo que incluye la red de orientación y tiempos de reacción explicó el $20 \%$ de la propensión a cometer errores en la conducción. Una alta orientación está asociada con una baja propensión a cometer errores, y los tiempos de reacción más lentos están relacionados con altos errores de conducción. Los resultados son consistentes con estudios previos y aportan nueva evidencia sobre el rol de los tiempos de reacción y redes atencionales en interacción con variables sociodemográficas y experticia sobre la propensión a cometer errores en la conducción. 
Palabras clave: Redes atencionales, Propensión al error, Conducción, Tránsito, Atención ejecutiva.

\begin{abstract}
Traffic accidents are a complex phenomenon resulting from a combination of environmental, vehicular and human factors, which have become one of the leading causes of death worldwide. Inattention is one of the main factors contributing to traffic accidents. The aim was to analyze the relationships between attention and the error proneness while driving. Posner's model states three attentional networks quantified by reaction time measures: orienting, alerting, and executive control (Posner, 1994; Fan et al., 2002). Orienting is responsible for the information selection. Alerting facilitates achieving and sustaining an alert state. Executive attention controls interference and solves conflicts between possible responses. Driver inattention was conceptualized from a perspective of individual differences as a "tendency or personal propensity of drivers to experience attentional lapses" (Ledesma et al., 2010, 2015). This tendency can be expressed at different levels of driving behavior: operational level, maneuvering, and strategic level (Michon, 1985). The sample consisted of 70 drivers from Buenos Aires (Argentina), both genders ( $57 \%$ female; $M_{\mathrm{age}}=29.29 ; S D=$ 9.258; $M$ experience years $=9.83 ; S D=8.861)$, inclusion criteria: driver's license, regular driving during the last two months (at least once a week), normal vision, and at least one year of driving experience. Factorial design 2 (low- high for each of the attentional networks) x 2 (gender). Measures: ARDES-ERIC (Ledesma et al., 2010): a 19-items self-report instrument to evaluate individual differences in the propensity to commit attentional failures while driving and can be classified according to the driving task level at which they occur (navigation, maneuvering, or control) (Alpha: .88; navigation Alpha: .744, maneuvering Alpha: .727, and control A1pha: .770), Attention Network Test (Fan et al., 2002) to measure three attentional networks: alerting (Alpha: .52), orienting (Alpha: .61), and executive attention (Alpha: .77) and RT attention
\end{abstract}

(Alpha: .87) and a sociodemographic questionnaire that includes question about driver behavior (e.g. frequency and experience). Results show that no relationship was detected between ARDES and age but there are significant correlation between ARDES and driving task level with Global Reaction Time (Global RT). ANOVA results show a significant interaction between Global Reaction Times and expertise on driving errors $\left[F_{(1,64)}=7.746 ; p<.01 ; \eta^{2}=\right.$ .108]. Experts drivers with low RT (lower processing speed) have a higher propensity to commit attentional failures while driving $\left(M_{\text {low }} R T=\right.$ 35.58; $\left.S D=13.08 ; M_{\text {high }} R T=26.95 ; S D=5.21\right)$. There are no interactions between Global RT, sociodemographics variables (age, gender), and driving frequency on propensity to commit errors. Global $R T$ correlates significantly with total score driving errors $(r=.373, p<.01)$. Executive Attention has a significant effect on total driving errors $\left[F_{(1,66)}=3.760 ; p=.05 ; \eta^{2}=.054\right]$, and only on the Control Dimension $\left[F_{(1,66)}=\right.$ $\left.7.889 ; p<.01 ; \eta^{2}=.124\right]$. There are no effects of Alerting and Orienting on total driving errors neither on each dimension of driving. A linear regression model involving the Orientation network and Global $R T$ explained the $20 \%$ of the total variance of the error proneness while driving $\left(\mathrm{R}^{2}\right.$ adjusted $\left.=.203\right)$. A higher level of Orienting attention is related to a lower propensity to commit errors $(\beta=-.332 ; p<.01)$, and a lower processing speed (higher Global RT) explained higher driving errors $(\beta=.242 ; p<$ $.05)$. Results are consistent with previous studies (López-Ramón et al., 2011) and provide new evidence about the role of executive control on specific dimensions of driving. In addition, the findings provide new evidence on the role of reaction times and attentional networks, in interaction with sociodemographic variables and expertise on the propensity to commit errors while driving. Limitations and theoretical-practical implications will be discussed.

Keywords: Attentional networks, Error proneness, Drive, Traffic, Executive attention. 


\section{Introducción}

El uso masivo de automóviles y otros vehículos a motor ha transformado al tránsito en una de las principales amenazas para la salud pública. Según la Organización Mundial de la Salud (OMS, 2009) cada año mueren en el mundo más de un millón doscientas mil personas como consecuencia de choques y otros incidentes de tránsito. Los accidentes de tránsito son efecto de la interacción de factores ambientales, vehiculares y humanos. En Argentina durante el período 2004-2011 se determinó que de los accidentes reportados, el $88 \%$ son a causa del factor humano (Centro de Experimentación y Salud Vial - CESVI, s.f.).

Diversos trabajos exploraron la relación entre la participación en accidentes, y la presencia o ausencia de factores permanentes del individuo (por ejemplo, percepción visual, atención selectiva, tiempos de reacción). Específicamente, algunos autores encontraron una relación significativa entre fallas cognitivas e incidentes automovilísticos (Larson, Alderton, Neideffer, \& Underhill, 1997; Wallace \& Vodanovich, 2003). Según Allahyari et al. (2008) los puntajes en las fallas cognitivas son fuertes predictores de errores durante la conducción en conductores.

\section{Procesos cognitivos en la conducta de manejo}

La diversidad de estímulos (internos externos) que recibimos compiten entre sí para hacer uso de los recursos limitados de nuestro sistema cognitivo. Posner (1980, 1994) propuso una teoría integradora en la cual la variedad de manifestaciones atencionales se produce en sistemas funcionalmente diferentes que interactúan entre sí. Estudios de neuroimagen han demostrado que una amplia variedad de tareas cognitivas puede ser vista como la activación de un conjunto distribuido de áreas neurales, cada una asociada con operaciones mentales específicas y además a neurotransmisores pri- marios específicos (Norepinefrina, Dopamina y Acetilcolina) (Posner, 1994). La evidencia de la neurociencia cognitiva plantea que la atención es un sistema complejo que incluye al menos, tres redes atencionales: alerta, orientación y atención ejecutiva (Posner \& Petersen, 1990; Wang \& Fan, 2007). La red de alerta refiere a un sistema que logra y mantiene un estado de detección de nuevos estímulos, que incluye la capacidad de aumentar la vigilancia frente a un estímulo inminente (Posner \& Petersen, 1990). Mientras la alerta intrínseca (tónica) se define como el control cognitivo de la vigilia y arousal, la alerta fásica prepara al sistema para dar respuesta a un estímulo critico (Fan \& Posner, 2004; Luna, Marino, Macbeth, \& Torres, 2016). Por otro lado, la red de orientación permite la selección de información desde el input sensorial tanto voluntaria (cuando la persona busca el objetivo dentro de su campo visual), como involuntaria (cuando la persona dirige su atención a un estímulo repentino). La red de atención ejecutiva o control ejecutivo es responsable de resolver el conflicto entre respuestas que compiten entre sí, y está involucrada en operaciones complejas como la planificación, toma de decisiones, detección de error, respuestas nuevas o no bien aprendidas (Fan, McCandiss, Summer, Raz \& Posner, 2002).

Un ejemplo cotidiano de la capacidad limitada del ser humano para procesar la información es la conducción (Groeger, 2002). La acción de conducir involucra un esfuerzo tanto físico como cognitivo, es decir, es una conducta compleja y requiere la ejecución de varias tareas al mismo tiempo. Desde una teoría de la arquitectura cognitiva humana se plantea la existencia de un recurso atencional limitado, focalizado sobre una meta actual que incrementa la accesibilidad al conocimiento relevante a la meta, en detrimento de otro tipo de conocimiento (Lovett, Reder, \& Lebiere, 1999). Este recurso limitado se distribuye cuando las tareas son muy demandantes cognitivamente, perjudicando la recuperación de la información relevante a la meta. Existen dife- 
rencias individuales de esta capacidad atencional, variando así la habilidad para acceder a la información relevante a la meta (Lovett, Reder, \& Lebiere, 1999). Al respecto Castro, Durán y Cantón (2006) señalan también que los límites de los recursos atencionales se evidencian en la medida en que una tarea requiera mayor atención, ya que ésta quedará menos disponible para otras tareas. Entonces, dividir la atención entre tareas complejas se dificulta más cuando estas tareas son similares, altamente demandantes y requieren atención continua; por eso el desempeño de una o de ambas se deteriorará inevitablemente (Victor, Engström, \& Harbluk, 2008).

Allahyari et al. (2008) examinaron la relación entre fallos cognitivos, errores de conducción y los datos de accidentes en conductores profesionales de Irán encontrando una correlación entre las puntuaciones totales del Cuestionario de Fracasos Cognitivos (CFQ) con las tasas de error de conducción, pero no con los accidentes. Posteriormente, Weaver, Bédarda, McAuliffe, y Parkkari (2009) utilizaron la prueba de Redes Atencionales (ANT) (Fan, McCandliss, Sommer, Raz, \& Posner, 2002) que mide redes atencionales, y el Test de Campo de Visión útil (UFOV) (Clay et al., 2005), una prueba cognitiva que pone a prueba simultáneamente el procesamiento de la información, tanto del centro como de la periferia del campo visual de conducción. Estos autores demostraron que ANT era altamente predictivo de la medida de UFOV, y junto a la puntuación global del ANT resultaron ser un factor predictivo de la capacidad de conducción. Sin embargo, el estudio de las relaciones entre medidas como el ANT y los errores en la conducción, aún requiere de mayor profundización en general, y especialmente en Argentina. Estos autores plantean la necesidad de mayor evidencia que respalde la predicción de otras conductas o respuestas que se dan durante la conducción (por ejemplo, la exploración), a partir de pruebas atencionales como el ANT (Weaver et al., 2009).

Por otra parte, Castro, Crundall, Chapman, Trawley y Underwood (2009) estu- diaron la interacción de las tres redes atencionales y el rol de la experiencia en la conducción vehicular. Demostraron que el patrón de resultados de los conductores no experimentados era diferente del que presentaban los conductores experimentados en el desempeño de la tarea atencional (ANTI) desarrollada por Callejas, Lupiañez y Tudela (2004). Es decir, los conductores sin experiencia tenían una diferencia funcional en las tres redes atencionales, pero en conductores con experiencia solo se encontraron patrones suaves de interacción entre las tres redes atencionales.

En el estudio de Mizobuchi, Chignell y Canell (2013) acerca de las diferencias individuales en conducción relacionadas con la multitarea, demostraron que personas con mayor capacidad cognitiva (atención y memoria de trabajo) pueden prestar más atención a una tarea primaria, sin afectar negativamente su desempeño en la tarea secundaria. Asimismo, las personas con baja conciencia de riesgo tienden a ser más distraídos por la tarea secundaria y quienes tienen una mayor conciencia de los riesgos, son más propensos a mantener su enfoque en la tarea primaria. Estas tareas secundarias tienden a ser más molestas para las personas con capacidades cognitivas inferiores; así, los que tienen una mayor conciencia de los riesgos, son más propensos a mantener su enfoque en la tarea primaria (Mizobuchi, Chignell, \& Canell, 2013).

\section{Propensión a cometer errores durante la conducción}

Cuando hablamos de un error humano nos referimos a un acontecimiento desfavorable condicionado por la acción de quien o quienes participan directa o indirectamente en su realización y control. Sobre esto, Montes (2013) señala que se entiende que los errores de inatención no solo se deben a variables situacionales o del tránsito, sino que pueden comprenderse a partir de factores más generales de propensión psicológica personal. Por lo tanto, cuando una persona es más propensa que otra a cometer 
ciertos errores atencionales significa que tiende a experimentar más accidentes que otros individuos con características sociodemográficas similares (e.g., edad, género, lugar de residencia), y con características o rasgos de personalidad estables (Visser, Pijl, Stolk, Neeleman, \& Rosmalen, 2007).

Según af Wahlberg y Dorn (2009), la propensión al error empieza a emplearse a inicios del siglo XX, en el momento que observaron que algunas personas participaban en mayor cantidad de accidentes que otras; en principio se referían a accidentes ocurridos principalmente en el ámbito laboral y posteriormente se utilizó para el área del tránsito. Los errores relacionados con la inatención son aquellos errores no intencionales o equivocaciones no relacionadas con falta de conocimiento o experiencia en la tarea, sino resultantes (total o parcialmente) de fallas atencionales, desviaciones no premeditadas de un plan (Reason, 1990). Son errores realizados de manera automática, alejándose de otros comportamientos que se ejecutan intencionadamente como las contravenciones a las normas de tránsito. Dichos errores se pueden clasificar en deslices (fallas atencionales), lapsus (fallas de memoria) y equivocaciones (Reason, 1990).

Por lo tanto, algunos conductores experimentarán con mayor intensidad o frecuencia que otros, este tipo de fallas, y estas variaciones entre conductores serán justificadas desde factores personales y no por factores situacionales o externos (distractores). Además, las diferencias entre conductores expresan un patrón o disposición más general de inatención en el funcionamiento de la vida diaria (Montes, 2013).

Para medir las diferencias individuales en errores de conducción como resultado de fallas de atención, Ledesma, Montes, Poó, \& López-Ramón (2010) elaboraron y validaron un cuestionario (Escala de errores relacionados con atención y los errores de conducción - ARDES/ERIC) y demostraron que los errores de conducción están fuertemente asociados con la propensión general de error, es decir, la falta de atención al re- alizar las actividades cotidianas. Este mismo cuestionario fue utilizado por LópezRamón y colaboradores (2011) para estudiar la relación entre edad, propensión personal a cometer errores relacionados con la atención durante la conducción y funcionamiento de las tres redes atencionales medidas con el test de redes atencionales para interacciones (ANTI; Callejas et al., 2004). Los resultados indicaron que los participantes con las puntuaciones más altas ARDES mostraron tiempos de reacción (TR) general más lentos y puntajes más altos en el estado de alerta fásica; los participantes de más edad mostraron una disminución en el rendimiento, menos preparación endógena para las señales de advertencia de alta prioridad y pobre control cognitivo en la resolución de conflicto, en comparación con los conductores más jóvenes.

El sistema neurocognitivo de atención de los conductores que explica los lapsos de atención durante la conducción está asociado positivamente con fallas cognitivas (Roca, Lupiáñez, López-Ramón, \& Castro, 2013). Además, los lapsos de atención se asocian negativamente con la vigilancia y de forma positiva con la insuficiencia cognitiva (Roca et al., 2013). Estos resultados apoyaron la idea de que el factor de lapsos del Cuestionario de Comportamiento del Conductor (DBQ) puede estar relacionado con la falta de atención durante la conducción: los participantes de su estudio que tienen más lapsos atencionales mientras conducen, tienden a ser los que tienen peor desempeño de vigilancia y mayor frecuencia de fallas cognitivas auto-reportados en la vida cotidiana. Montes (2013) por su parte, incorpora una tarea experimental de atención selectiva, la de búsqueda visual conjunta (BVC), para analizar el grado de convergencia con los puntajes obtenidos en la escala de autoinforme ARDES.

La tendencia personal a experimentar errores atencionales puede manifestarse en todos o en alguno de los niveles de la tarea de conducir, desde los más automatizados a aquellos que requieren un mayor control atencional (Ledesma, Montes, Poó, \& Ló- 
pez-Ramón, 2015). Michon (1985) propone que las tareas del conductor están divididas en tres niveles: (1) un nivel estratégico que es el planeamiento general del viaje e incluye elección de metas de viaje (ahorrar tiempo, evitar el tráfico), selección de rutas y evaluación de costos y riesgos implicados en cada opción; (2) un nivel táctico, momento mismo de la conducción, donde se realizan las maniobras y consiste en una negociación permanente con las situaciones que surgen en el momento (decidir bajar la velocidad frente a una señal de tránsito, evitar obstáculos, adelantarse) y (3) un nivel operacional que se refiere al control real del vehículo (control de posición lateral y longitudinal, ajustes de velocidad) que en general, constituyen patrones de acción automáticos.

Otra diferencia entre niveles es la escala temporal en la que se desarrollan: mientras el nivel operacional abarca apenas unos milisegundos, el nivel de maniobra se desarrolla en una escala de segundos y el nivel estratégico implica una duración mayor (Montes, 2013).

En síntesis, estudios previos brindan evidencia sobre algunos procesos cognitivos claves involucrados tanto en la conducción como en los errores que se cometen durante la misma, los cuales han sido estudiados en diferentes contextos socioculturales. En aras de evitar accidentes relacionados con la inatención, estos autores sugieren que se continúen los estudios sobre la propensión a cometer errores como una característica individual relacionada con la falta de atención y específicamente con las redes atencionales.

El presente estudio se propuso identificar las interrelaciones entre las redes atencionales (orientación, alerta y control ejecutivo) y características sociodemográficas en la propensión a cometer errores relacionados con la inatención en conductores de vehículos a motor. Conocer el rol específico de las redes atencionales junto a otros factores como los sociodemográficos y la experticia en la tarea de conducción, es relevante no solo por su aporte teórico, sino también por la posibilidad de predecir la propensión a cometer errores relacionados con la inatención durante la conducción. Esto podría orientar la implementación de políticas de seguridad vial y programas de detección de diferencias individuales mucho más precisos para prevenir accidentes de tránsito.

\section{Pregunta de investigación e hipótesis del presente estudio}

¿De qué manera se relacionan las tres redes atencionales y el Tiempo de Reacción total (TR total) con la propensión a cometer errores en la conducción de vehículos a motor? ¿Sobre qué dimensión específica de la propensión (maniobra, control, navegación) influyen cada una de las tres redes atencionales?

Las características sociodemográficas (sexo, edad y experticia) ¿moderan la influencia de algunos de estos componentes atencionales sobre la propensión a cometer errores durante la conducción?

¿En qué medida la propensión a cometer errores explica dichas variables de atención y sociodemográficas?

Dado el carácter automático y no intencional de los errores en la conducción, relacionados con la inatención (Reason, 1990) y los lentos TR que se han encontrado en conductores que más errores cometen $(\mathrm{Ca}-$ llejas et al., 2004), se hipotetiza que aquellos conductores con TR lentos en las tres redes atencionales presentarán una mayor cantidad de errores en la conducción (Montes, 2013; Roca et al., 2013; Weaver et al., 2009). Específicamente, sujetos con tiempos de reacción altos (más lentos) en la red de alerta serán más propensos a cometer errores de inatención durante la conducción. Además, el TR total influirá significativamente sobre la dimensión de maniobra debido a que ésta involucra una negociación permanente frente a situaciones que surgen en el momento mismo de la conducción (Michon, 1985). Solo la dimensión de maniobra se ha asociado con la partici- 
pación en choques y multas de tráfico (Ledesma et al., 2015), y por lo tanto, se especula que el TR total no tendrá efecto sobre otras dimensiones de la propensión a cometer errores.

Se plantea como hipótesis de estudio que el sexo y la edad no moderarán la relación entre redes atencionales y errores en la propensión a cometer errores, dado que se han hallado divergencias en cuanto a las diferencias en TR total según sexo (Luna et al., 2016; Miró, Martínez, Sánchez, Prados \& Lupiáñez, 2014) y según edad (Ledesma, Montes, Poó, \& López-Ramón, 2010; López-Ramón et al., 2011). En cambio, la experticia moderará la influencia de las redes atencionales y el TR total sobre la cantidad de errores, debido al funcionamiento diferencial de las tres redes entre conductores experimentados y no experimentados (Castro, Crundall, Chapman, Trawley \& Underwood, 2009).

Por último, se espera encontrar que diferencias individuales en las tres redes atencionales, junto a variables como la experticia, expliquen la propensión a cometer errores atencionales en la conducción.

\section{Método}

\section{Participantes}

Se seleccionó una muestra intencional de 70 personas de distinto género $(57 \% \mathrm{mu}-$ jeres) y edades comprendidas entre los $19 \mathrm{y}$ 59 años $(M=29.29 ; D S=9.258)$. Como criterios de inclusión se consideraron: ser mayor de edad, tener registro de conducir y haber conducido regularmente durante los últimos dos meses (al menos una vez por semana) con un mínimo de experiencia de un año. Además, debían reportar visión normal o corregida, a normal. Los participantes indicaron una frecuencia de manejo promedio de 4.29 veces por semana $(D S=2.24)$ y un promedio de 9.83 años de experiencia $(D S=8.861)$.

\section{Instrumentos}

\section{Test de Redes Atencionales (ANT) (Fan, McCandliss, Sommer, Raz, \& Pos- ner, 2002).}

Este test mide tres redes atencionales: Alerta, Orientación y Atención Ejecutiva. Se le pide al postulante que determine la dirección de una flecha central (hacia la izquierda o hacia la derecha). El experimento total demora unos 20 minutos aproximadamente y contiene cuatro bloques: un primer bloque de práctica de dos minutos (con 24 ensayos que incluyen feedback) y tres bloques experimentales de 5 minutos cada uno sin feedback. Cada bloque experimental consiste en 96 ensayos: 4 condiciones de señal (doble señal, sin señal, señal centrada, señal espacial) x 2 posiciones de señal (arriba-abajo) x 2 direcciones de flechas (izquierda-derecha) x 3 condiciones objetivo (neutral, congruente e incongruente) $x$ 2 repeticiones (Fan et al., 2002). Se registran los tiempos de reacción medidos en milisegundos. Una mediana de TR es computada para cada una de las 12 combinaciones de condiciones de señal por condiciones objetivo (utilizando solo los ensayos correctos). Luego se calculan las medias de las medianas de tiempos de reacción para cada una de las cuatro condiciones de señal (promediando a través de las tres condiciones objetivo) y para las condiciones congruentes e incongruentes (a través de las cuatro condiciones de señal). Finalmente, se estima la eficiencia de las tres redes atencionales de la siguiente forma: Alerta (media de TR sin señal - media de TR con doble señal), Orientación (media de TR señal central - media de TR señal espacial) y Atención Ejecutiva (media de TR incongruentes - media de TR congruentes). Fan et al. (2002) han informado respecto de la confiabilidad test re-test los siguientes coeficientes: $r=.87$ para la medida de tiempos de reacción total; $r=.52$ para la red de alerta; $r=.77$ para la atención ejecutiva y $r=.61$ para la red de orientación. 
ARDES - ERIC (Cuestionario de experiencias durante la conducción) (Ledesma, Montes, Poó, \& López-Ramón, 2015).

Se trata de un instrumento de autoinforme que mide la propensión a cometer errores relacionados con la inatención en conductores. Consiste en una escala tipo Likert de 5 puntos, desde nunca o casi nunca (1) a siempre o casi siempre (5), compuesta por 19 ítems que evalúan diferencias individuales en la propensión a cometer errores de desempeño en la conducción, que resultan total o parcialmente de la inatención. Los puntajes altos en la escala reflejan alta propensión al error. Ejemplos de ítems: "De pronto, notar que he perdido o equivocado el camino en un trayecto que conozco", "Al llegar a una esquina, no darme cuenta de que un peatón está cruzando la ca1le". Los ítems son agrupados de acuerdo a la complejidad o nivel de la tarea en: (a) Control, el cual implica la ejecución de acciones básicas, como la dirección, los frenos y manipulación de otros controles del automóvil, es decir que forman parte de la rutina automática y sin esfuerzo al operar un vehículo (ítems 2, 10, 14, 15, 18); (b) Maniobra: este nivel es un proceso que incluye más al medio ambiente; implica procesamientos controlados y comportamientos de conducción tales como cambiar de carril y cruzar una intersección; los errores a este nivel pueden tener más consecuencias drásticas sobre el rendimiento de conducción y aumentar la probabilidad de colisiones de vehículos de motor (ítems 3, 5, 6, 7, 8, 9, $13,17) ;$ y (c) Navegación, que es la tarea de conducción de nivel superior y se ocupa de los procesos tales como la planificación y mantenimiento de rutas (ítems 1, 4, 11, 12, 16). En la muestra del presente estudio, ARDES presenta una alta consistencia interna $(\alpha=.874)$, resultado similar al reportado por Ledesma et al. (2010) $(\alpha=.88)$, siendo también adecuada la confiabilidad de cada una de las subescalas o dimensiones (.770 para Control, .727 para Maniobras y .744 para Navegación), valores similares a los reportados por Ledesma y colaboradores (2015).

\section{Cuestionario sociodemográfico.}

Se administró un cuestionario que recaba información acerca de algunos datos sociodemográficos (género, edad y nivel educacional) y de conducción (frecuencia y años de manejo).

\section{Procedimiento}

Procedimiento de recolección de datos.

Los sujetos fueron invitados a participar del estudio de forma voluntaria, garantizando el anonimato y la confidencialidad en la administración de los instrumentos y en el tratamiento de los datos; dejaron constancia con su firma en el consentimiento informado. Para los cuestionarios sociodemográfico y de experiencias durante la conducción (ARDES), se utilizó Google Drive Forms. La tarea atencional ANT se realizó a través del software E-Prime con una computadora portátil HP-Sleekbook 14" de pantalla a color. Las respuestas se recopilaron mediante mouse periférico ubicado en el medio, entre el participante y la computadora, y centrado de acuerdo a la pantalla. Se le informó al participante las consignas de la tarea oral y escrita, a la vez que fueron instruidos para responder a la dirección del estímulo objetivo (es decir, la flecha central) utilizando los pulgares, derecho cuando la flecha central estaba apuntando hacia la derecha, e izquierda cuando la flecha central apuntaba al lado izquierdo de la pantalla. El participante debió estar sentado a $60 \mathrm{~cm}$ de la pantalla de la computadora y apagar su celular. Fue administrado de forma individual, en un espacio cerrado, en silencio y sin distracciones.

\section{Procedimiento de análisis de datos.}

Se usó el valor de la mediana de la edad $(M d n=26)$ para dividir a los participantes en dos grupos: jóvenes y adultos. Los 33 conductores jóvenes (17 hombres, $16 \mathrm{mu}$ jeres) fueron incluidos en el grupo Edad 1 (rango: $19-25$ años; $M=22.52$ años; $D S=$ 
2.138 ). Los 37 conductores adultos ( $24 \mathrm{mu}-$ jeres, 13 hombres) conformaron el grupo Edad 2 (rango: 26-59 años; $M=35.32$ años; $D S=8.98)$. Se clasificó a los conductores en dos grupos según su grado de experticia (años de experiencia y frecuencia de manejo): 42 conductores expertos y 28 conductores novatos. Para ello se armaron dos grupos de acuerdo a los años de experiencia (Bajo vs Alto) utilizando como punto de corte la mediana $(M d n=3.5)$. Se consideró experto si la frecuencia era Alta y los años de experiencia mayor o igual a cinco años en caso contrario eran novatos; pero si la frecuencia era Baja y los años de experiencia era mayor o igual a ocho años entonces, se los consideró expertos, caso contrario formaban parte del grupo de novatos.

Se establecieron niveles para las tres redes atencionales de acuerdo a las medianas para las distribuciones de cada red: para Alerta los valores por debajo de la mediana de $39.17 \mathrm{~ms}$ fueron considerados pertenecientes al nivel Alto, mientras que valores por encima de ese valor pertenecían al nivel Bajo. Para el nivel de Orientación los valores por debajo de la mediana de $42.17 \mathrm{~ms}$ pertenecían al nivel Alto, caso contrario al nivel Bajo. Los valores de Atención ejecutiva por debajo de la mediana de $103.19 \mathrm{~ms}$ correspondían a un nivel Alto, mientras que para los que estaban por encima fueron del nivel Bajo. Se calcularon los logaritmos naturales (ln) de los tiempos de reacción total de las tres redes atencionales: los valores por encima de 6.40 fueron considerados del grupo de TR lentos y en el caso de los valores por debajo de la mediana se agruparon en TR rápidos.

Se utilizó IBM SPSS STATISTICS 21 para el análisis de los datos estadísticos. Se calcularon las medidas descriptivas de las distintas variables, para luego conformar los grupos (porcentajes, tablas de frecuencia, medias y desvíos estándar). Se exploraron las correlaciones entre las variables usando correlación bivariada de Pearson. Se realizó un análisis clásico de medidas repetidas intrasujetos de la prueba ANT (4 condiciones de señal: doble señal, sin señal, señal centrada, señal espacial x 3 condiciones de flecha u objetivo: central, congruente e incongruente) (Fan et al., 2002). Se incluyó además la variable género como factor intersujeto para estudiar la interacción con las condiciones y objetivo. Se repitió este análisis con la variable edad, experticia y nivel de errores de conducción del ARDES, como factores intersujetos por separado, porque no se contaba con la mínima cantidad de sujetos por celda para realizar un análisis factorial completo. Por otro lado, siguiendo la metodología empleada por Weaver et. al. (2009), se llevaron a cabo Análisis Univariados de Variancia utilizando como variables independientes los dos niveles de cada red atencional y del TR total, sobre las medidas dependientes del ARDES general. Además, se consideraron cada una de las dimensiones del ARDES como medidas dependientes realizando un Análisis Multivariado de Variancia (MANOVA). Para estudiar los efectos de interacción se llevó a cabo un ANOVA de 2 Grupos de TR total (TR lento / TR rápido) x 2 de Experticia (Experto/Novato) sobre ARDES total. Lo mismo se realizó para estudiar la interacción de la edad con TR total: 2 Grupos de Edad (Edad 1 / Edad 2) x 2 de TR total (Bajo/Alto) por un lado, y la interacción del género con TR total: 2 grupos de Sexo (Femenino/Masculino) x 2 de TR total (Bajo/Alto). Finalmente, se realizó un análisis de regresión lineal múltiple, con el método de pasos sucesivos, para estudiar qué variables individuales explican -y en qué medida- el puntaje ARDES.

\section{Resultados}

\section{Medidas descriptivas}

En la Tabla 1 se resumen los estadísticos descriptivos de los TR (en milisegundos) de las distintas condiciones de señales y de flechas (1a) y las precisiones (1b). Los análisis muestran que los tiempos de reacción son más rápidos cuando hay señales espaciales $(M=544)$ que cuando no se presentan 
señales $(M=620)$. Además, en las condiciones incongruentes aumentan los TR (mayor demora), especialmente cuando no hay una señal. La precisión desciende también en estas condiciones de incongruencia. Las medias de eficiencia de cada red atencional fueron: $40.96 \mathrm{~ms}(D S=30.61)$ para alerta, en el caso de la red de Orientación fue de $44.24 \mathrm{~ms}(D S=42.17)$ y para la red de Atención ejecutiva la media fue de $107.90 \mathrm{~ms}$ $(D S=44.90)$.

\section{Redes atencionales, edad y propensión a cometer errores: correlaciones}

En la Tabla 2 se observan las correlaciones entre cada una de las redes atencionales y TR Total con el puntaje global y dimensiones de la escala ARDES. Se observa que el puntaje total de esta escala correlacionó de manera positiva y significativa con la variable TR Total, aunque la fuerza de esta correlación es débil ( $r=.373 ; p=.002)$. De esta manera, a nivel general se observa que, a más altos TR (más lentos), mayor propensión a cometer errores por inatención.

Si bien no se observó una correlación entre la edad y la escala ARDES, existe una correlación positiva y muy significativa entre edad y TR Total $(r=.481, p<.001)$ de tal forma que a mayor edad, más largos los TR o más lentos en su procesamiento general de las redes atencionales.

\section{Atención y propensión a cometer errores: efectos principales e interacciones}

Los resultados del ANOVA intrasujeto señalaron un efecto de interacción entre la condición de señal y objetivo, indicando cierta falta de independencia entre las redes $\left(F=2.802 ; p=.019 ; \eta^{2}=.041\right)$. Además, se halló un efecto significativo de interacción entre condición de señal, objetivo y género sugiriendo que las mujeres son más rápidas en sus TR que los varones cuando se presenta el estímulo incongruente solo en condiciones de señal central $(F=2.338 ; p=.045$; $\eta^{2}=.034 ; M_{\text {mujeres }}=680.38 \mathrm{~ms} ; M_{\text {varones }}=$ $710.41 \mathrm{~ms} ; p=.07)$. La diferencia por género en cuanto a la precisión en dichas condiciones no resultó significativa $\left[F_{(1,68)}=\right.$ $.375 ; p=.542 ; M_{\text {mujeres }}=.91 ; M_{\text {varones }}=$ .93]. El ANOVA de medidas repetidas sobre la precisión en las distintas condiciones no encontró efectos de interacción significativos entre señal y objetivo, como así tampoco entre señal, objetivo y género.

No se halló un efecto de interacción entre señal, objetivo y experticia. Tampoco se encontró interacción entre señal, objetivo y grupos de edad. De la misma forma, no hubo interacción con niveles de errores de conducción del ARDES.

Cuando se analiza desde el punto de vista de la eficiencia de cada red, se encontró un efecto principal de la red atencional ejecutiva sobre la propensión a cometer errores, aunque la significación estadística fue marginal y el tamaño del efecto muy bajo $\left[F_{(1,66)}=3.760 ; p=.050 ; \eta^{2}=.054\right]$. De esta manera, los conductores con TR altos frente a estímulos incongruentes (más lentos en general) tienden a cometer mayor cantidad de errores por inatención durante el manejo, comparados con los conductores con TR más rápidos (ver Tabla 3). No se halló un efecto principal del nivel de alerta ni del nivel de orientación sobre la propensión a cometer errores.

Como se muestra en la Figura 1, se encontró un efecto de interacción significativo entre la experticia y el nivel de TR total en su efecto conjunto sobre la propensión a cometer errores $\left[F_{(1,64)}=7.746 ; p<.01\right.$; $\left.\eta^{2}=.108\right]$ (ver Tabla 4). Se analizó si había interacción entre nivel TR total y nivel de precisión de la tarea ANT y se encontró una interacción sobre la cantidad de errores de conducción reportados en la tarea ARDES $\left[F_{(1,47)}=11.342 ; p<.005 ; \eta^{2}=.194\right]$. Los conductores más lentos en sus TR y baja precisión en el ANT fueron los que reportaron mayor cantidad de errores de conducción ( $M_{\text {errores }}$ para bajo TR total y baja precisión: 43.86 ; $M_{\text {errores }}$ para bajo TR total y alta precisión: 28.53). No hubo diferencia en los errores de conducción entre conductores con baja y alta precisión mientras tenían tiempos de reacción rápidos. 
Los resultados indicaron que no se encontró interacción entre la edad y el TR total sobre la propensión a cometer errores. De la misma forma, no se halló una interacción significativa entre el sexo (Femenino y Masculino) y el TR total (TR Bajo y TR Alto) en la propensión a cometer errores (ver Tabla 4). No se encontró un efecto del nivel de TR Bajo-Alto sobre el puntaje total de la propensión a cometer errores durante la conducción.

Se realizaron MANOVAs considerando dos niveles de eficiencia para cada red atencional (Alerta, Orientación y Atención Ejecutiva) como medidas independientes y los puntajes de las tres subescalas ARDES (Maniobra, Control y Navegación) como medidas dependientes. Los resultados indicaron que existe un efecto significativo de la Atención Ejecutiva sobre la subescala Control de propensión a cometer errores durante la conducción $\left[F_{(1,66)}=7.889 ; p<.01\right.$; $\left.\eta^{2}=.124\right]$. En cambio, no se encontró un efecto de la Atención Ejecutiva sobre $\mathrm{Na}$ vegación y Maniobra. Tampoco se hallaron efectos significativos de Orientación, Alerta y TR total sobre ninguna de las tres dimensiones de la escala ARDES (ver Tabla 5).

\section{Variables explicativas de la propensión a cometer errores}

Con el fin de analizar si era posible predecir la propensión a cometer errores por inatención a partir de las variables atencionales y sociodemográficas consideradas en esta investigación, se llevó a cabo un análisis de regresión lineal múltiple por pasos sucesivos. En el modelo se incluyó como variable dependiente el puntaje total de ARDES y como variables independientes las medidas de las tres redes atencionales, TR Total, edad, género y experticia. Solamente las variables de la red de Orientación y la medida de TR Total fueron significativas y explicaron el $20 \%$ de la variancia total de la propensión a cometer errores por inatención en la conducción $\left(R^{2}\right.$ ajustado= .203 ), excluyendo las otras variables. La variable de mayor peso fue la red de orien- tación $(\beta=-.332 ; p=.007)$ de tal forma que a más altos tiempos de reacción (más lentos), menor cantidad de errores, y viceversa. La velocidad general de las tres redes atencionales mostró, en cambio, un menor valor explicativo y en sentido positivo $(\beta=.242$; $p=.045)$ : una menor velocidad de procesamiento general predice una mayor cantidad de errores en la conducción.

\section{Discusión}

En este estudio se preguntó en primer lugar, sobre cómo se relacionan las redes atencionales y el Tiempo de Reacción total (TR Total) con la propensión a cometer errores en la conducción de vehículos a motor. Los análisis por separado de las redes atencionales mostraron que solo hay un efecto de la red de Atención Ejecutiva sobre la propensión a cometer errores. Por lo tanto, sujetos con mayores dificultades a la hora de controlar estímulos prepotentes que interfieren, tienden a una mayor propensión a cometer errores durante la conducción. Si bien nuestra hipótesis inicial esperaba que la red de alerta tuviera un efecto sobre la propensión a cometer errores, los resultados indican que éstos pueden deberse a diferencias individuales en la atención ejecutiva. La red de atención ejecutiva refiere a la supervisión y resolución de conflictos en la planificación, toma de decisiones, detección de errores, y superación de acciones habituales tal como señalan Wang y Fan (2007). Es decir, se encuentra relacionada con las actividades desempeñadas durante la tarea, mientras que la alerta se refiere a la función de lograr y mantener un estado de preparación para los próximos eventos relacionados con la tarea (Fan \& Posner, 2004). Este hallazgo está relacionado con el efecto específico que se encontró de la atención ejecutiva sobre el aspecto del control durante la conducción. Estos resultados son congruentes con la función definida por la atención ejecutiva, que "implica mecanismos de seguimiento y resolución de conflicto entre los pensamientos, 
sentimientos y respuestas" (Posner \& Rothbart, 2007, p. 7). Es lógico pensar que aquellos conductores con mayores dificultades atencionales en este sentido, tenderían a cometer más errores en las funciones de control: ejecución de las acciones básicas como la dirección, los frenos y manipulación de otros controles de automóviles. En este tipo de situaciones el conductor cuenta con un tiempo limitado para enfrentar o evitar el peligro.

La segunda pregunta del presente trabajo se refiere a la participación de variables sociodemográficas (sexo, edad) y experticia como moderadoras de la influencia de las redes atencionales sobre la propensión a cometer errores durante la conducción. Es necesario aclarar que no fue posible un análisis factorial completo para analizar todas las interacciones al mismo tiempo, debido a la pequeña muestra de sujetos. Los resultados indicaron, en primer lugar, que las mujeres tienen TR más rápidos que los varones cuando se presenta el estímulo incongruente solo en condiciones de señal central, manteniendo la precisión de la tarea. Sin embargo, no se halló una interacción entre los TR total y sexo ni un efecto principal del sexo sobre la cantidad de errores en la conducción. Estos hallazgos si bien son exploratorios están indicando un funcionamiento diferente en los tiempos de reacción en tareas viso-espaciales entre hombres y mujeres que son coherentes con estudios previos (Bradshaw \& Nettleton, 1983; Kolb \& Whishaw, 1985; Musso, 2016; Vaquero, Cardoso, Vázquez \& Gómez, 2004). Futuros estudios con una muestra más grande permitirían profundizar estos hallazgos.

Por otro lado, no se encontró un efecto de interacción entre TR total y edad sobre la propensión a cometer errores en la conducción. Sin embargo, existe una correlación positiva y significativa entre edad y TR total $(r=.481 ; p<.001)$. Estos hallazgos coinciden con otros (e.g. Ledesma et al., 2010; Montes, 2013) donde no se encontraron diferencias en la propensión a cometer errores de conducción según variables sociodemo- gráficas (edad y género). No obstante, la investigación de López-Ramón et al. (2011) reportó que la variable edad estaba relacionada con un aumento en TR generales en el sentido de que los participantes de mayor edad mostraron mayor TR en general (más lentos). Asimismo, observaron que existía un efecto significativo de la señal de alerta (ANTI) sobre el puntaje de propensión a cometer errores de conducción (ARDES), y de la señal de alerta y edad: así los participantes que reportaron mayor propensión a cometer errores durante la conducción y aquellos de mayor edad mostraron mayor alerta. Sin embargo, hay que tener en cuenta que en la investigación mencionada, el TR Total se trata como una medida general del desempeño que se ha moderado en relación con una medida directa de vigilancia utilizada en ANTI, aunque los mismos autores informan que no se considera un índice de vigilancia si es usado aisladamente.

Con respecto al efecto moderador de la experticia, se encontró un efecto de interacción entre el TR total y la experticia en su influencia conjunta sobre la propensión a cometer errores. De esta forma, los participantes expertos con tiempos de reacción más lentos tienen mayor propensión a cometer errores durante la conducción, a diferencia de los expertos con tiempos de reacción rápidos. Es decir, los conductores expertos más lentos tienden a reportar más errores de conducción quizás por factores de auto-regulación o sobre-confianza de su propia habilidad, cuestión que deberá ser indagada en futuros estudios. Investigaciones previas han demostrado que la propensión a cometer errores atencionales durante la conducción se relaciona con TR más lentos de tareas atencionales (López-Ramón et al., 2011; Montes, 2013). Por otro lado, los novatos con TR rápidos tienden a una mayor cantidad de errores de conducción que los expertos con TR rápidos, ya sea porque aún no está suficientemente automatizada la tarea o bien porque tienden a sobrestimar su experiencia. Se sabe que la atención es un recurso atencional limitado que frente a una tarea compleja es distribuida (Castro et al., 
2006; Lovett, Reder \& Lebiere, 1999). Con la práctica, tiempos de reacción general más rápidos ayudarían al novato a automatizar la tarea, cometiendo por lo tanto menos errores de conducción. Si bien estudios previos señalan diferencias en las tres redes atencionales entre conductores novatos y expertos (Castro et al., 2009), los resultados del presente trabajo apoyarían la hipótesis de que diferencias básicas en TR son las que contribuyen como base para que la experiencia y práctica tengan efecto sobre una menor propensión a cometer errores durante la conducción. Además, es importante tener en cuenta el resultado de interacción entre TR y precisión, ya que son los conductores con TR lentos e imprecisos quienes tienen mayor propensión a cometer errores. Esto implica que ambas medidas TR y precisión del ANT se refieren a aspectos diferentes del procesamiento cognitivo: un funcionamiento rápido del sistema cognitivo permitiría disminuir los errores mientras se conduce, aunque la precisión atencional sea baja. Posner (2008) ha confirmado que las medidas globales de rendimiento TR y precisión pueden reflejar diferentes estrategias para abordar la tarea.

Con respecto a la tercera pregunta de investigación, el modelo hallado con el análisis de regresión múltiple encontró que la red de orientación y el TR total son variables asociadas a la propensión a cometer errores durante la conducción. Para este modelo la red de orientación fue la variable de mayor peso explicativo en sentido negativo, de tal forma que a más lentos tiempos de reacción, menor cantidad de errores, y viceversa. Este resultado pareciera ser controvertido teniendo en cuenta los resultados anteriores. Los tiempos de reacción lentos en la red de orientación implicaría una dificultad para desplazar la atención de una señal a otra (Posner \& Petersen, 1990). Sin embargo, López-Ramón et al. (2011) han sugerido que los conductores con menor propensión a cometer errores podrían estar más involucrados con la tarea de conducir y, por lo tanto, tener mayor dificultad para desenganchar su atención de esta tarea a otros estímulos. Al contrario, conductores con mayor propensión a cometer errores pueden estar menos comprometidos con la tarea y, por lo tanto, son atraídos con mayor facilidad a una señal periférica. La red de orientación opera dirigiendo recursos atencionales, es decir, desplazando la atención, cuando se presenta una señal a través de la información sensorial. La conducción es una tarea compleja que demanda de nuestros recursos, y por lo tanto la red de orientación es necesaria para asignar selectivamente atención a los estímulos adecuados (e.g., la necesidad de orientar la atención hacia el auto que se encuentra adelante y los que salen de caminos laterales). Además, la red de orientación permite estar en un estado apropiado para ser capaz de procesar los eventos de alta prioridad durante la conducción (por ejemplo, cuando el conductor de adelante frena repentinamente) (LópezRamón et al., 2011).

Por otro lado, una menor velocidad de procesamiento general contribuye a explicar, aunque en menor medida, una mayor cantidad de errores en la conducción. Esto es consistente con el hallazgo de la interacción entre tiempos de reacción y experticia, según el cual los conductores expertos con una menor velocidad de procesamiento general presentaban mayor propensión a cometer errores durante la conducción. Al mismo tiempo, esto coincide con estudios previos (López-Ramón et al., 2011; Montes, 2013). Lellis et al. (2013) en su análisis de los estudios sobre orientación visual de la atención, encontraron que a través de medidas de TR se puede contribuir a una mejor comprensión de cómo el sistema nervioso selecciona información pertinente del medio ambiente y los circuitos neuronales implicados.

\section{Limitaciones y futuros estudios}

La falta de estudios previos sobre propensión a cometer errores relacionados con la inatención durante la conducción, sumado a que fueron realizados en ciudades específicas con muestras pequeñas, difi- 
cultó la comparación de los resultados obtenidos en el presente estudio. Si bien este trabajo tuvo una muestra más grande que las de investigaciones previas, se recomienda utilizar muestras de mayor tamaño y aleatorias, con el fin de aumentar la potencia observada e incrementar el tamaño de los efectos que se encontraron en el presente trabajo. Esto también permitiría profundizar los hallazgos respecto a las interacciones entre las múltiples variables consideradas en el presente estudio. En esta misma línea sería interesante poder comparar la interacción del nivel de atención con residencia (centros urbanos vs centros con baja densidad poblacional) en la propensión a cometer errores relacionados con la inatención, y con registros de accidentes $\mathrm{y} / \mathrm{o}$ infracciones de tránsito reales como variables dependientes.

Los resultados del análisis de regresión múltiple del presente trabajo deben considerarse como exploratorios, dada la poca cantidad de casos de la muestra. Futuros estudios podrían replicar dichos análisis por separado diferenciando grupos de expertos y novatos.

Otra cuestión a tener en cuenta, es que la tarea de ANT es una tarea atencional general que no fue diseñada para evaluar atención en situaciones específicas como el tránsito. Por ello, en los futuros estudios conviene adaptar la prueba al área utilizando estímulos del contexto vial (e.g., en situaciones simuladas de conducción que incluyan bocinas, señalización vial, semáforos, etc.). Si bien otros autores (Roca et al., 2013; Weaver, Bédard, \& McAuliffe, 2013) han desarrollado versiones de ANT que incluyen coches en lugar de flechas, dichos autores indican que el uso de estos estímulos más amigables no impactaría so- bre el tiempo requerido para completar la tarea. También hay que tomar en cuenta que en el presente trabajo las medidas dependientes pertenecen a una escala de autoinforme, que si bien tiene sus ventajas, también presenta riesgos (e.g., susceptibilidad frente a sesgos de respuesta, distorsiones de la memoria u olvidos, subestimaciones de los hechos). Sería importante que a futuro puedan realizarse estudios conjuntos con métodos experimentales, como por ejemplo, simuladores de conducción.

Se necesita considerar en futuros estudios, la complejidad de la tarea de conducir y por lo tanto, la mayor cantidad de variables a tener en cuenta cuando se trata de predecir la propensión a cometer errores en ella. Finalmente, se sabe que la realización simultánea de múltiples actividades complejas (eg., uso del celular, hablar con los pasajeros, conducir, fijar la visión, etc.) afecta al desempeño y produce errores. Por ello hay que tener en cuenta las limitaciones de los procesos atencionales para comprender el problema de los incidentes relacionados a la inatención.

Se espera que este trabajo impulse el desarrollo de líneas de investigación en el área de la psicología del tránsito, más específicamente, respecto del rol del factor psicológico en el error humano y en la siniestralidad vial. Además, se espera que los resultados sirvan para promover y mejorar programas de prevención y mitigación de fallas atencionales al conducir. Estos hallazgos podrían orientar a recortar la brecha entre el área de la investigación de procesos cognitivos básicos, el área de salud pública y de seguridad vial, en vista a una mayor concientización y prevención de los accidentes de tránsito. 
Redes atencionales y errores en la conducción

Tabla 1

Medias TR y precisión para cada condición

\begin{tabular}{ccccc}
\hline \multirow{2}{*}{ Congruencia } & \multicolumn{4}{c}{ Tipo de Señal } \\
\cline { 2 - 5 } & Ninguna & Central & Doble & Espacial \\
\hline (a) Medias TR (mseg) $y$ desviación estándar & & \\
Congruente & $620(90)$ & $590(97)$ & $568(89)$ & $544(88)$ \\
Incongruente & $733(140)$ & $705(131)$ & $694(125)$ & $649(120)$ \\
Neutral & $599(82)$ & $573(78)$ & $557(83)$ & $542(99)$ \\
(b) Precisión (\%) $y$ desviación estándar & & & \\
Congruente & $99(3)$ & $99(3)$ & $99(2)$ & $99(4)$ \\
Incongruente & $93(17)$ & $92(18)$ & $92(18)$ & $94(17)$ \\
Neutral & $99(5)$ & $98(5)$ & $99(3)$ & $99(4)$ \\
\hline
\end{tabular}

Tabla 2

Correlaciones de Pearson

\begin{tabular}{|c|c|c|c|c|c|c|c|c|c|}
\hline & I & 2 & 3 & 4 & 5 & b & 7 & 8 & 9 \\
\hline 1. Edad & & $4 \$ 1^{*}$ & 011 & 027 & Dil & -212 & -0.058 & -0.053 & 017 \\
\hline 2. TH & & & $34 "$ & $335^{\prime \prime}$ & $258^{\circ}$ & .044 & "34t" & .174 & $373^{-}$ \\
\hline 3. Conirol & & & & $610^{*}$ & hN" & 087 & -34 & -164 & 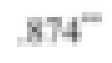 \\
\hline 4. Manioks & & & & & $4 \mathrm{kS}^{\mathrm{m}}$ & $0 x$ & $-407^{m}$ & $-10 s$ & $850^{\prime \prime}$ \\
\hline 9. Navepucion & & & & & & (M) & $-316^{\circ}$ & 10,074 & $\mathrm{Bub}^{-}$ \\
\hline b. Ahria & & & & & & & 192 & -114 & 840 \\
\hline 7. Oricetweiln & & & & & & & & 102 & $-459^{m}$ \\
\hline 8. Amrision Ejoutiva & & & & & & & & & -124 \\
\hline 4. ARDES & & & & & & & & & \\
\hline
\end{tabular}

** La correlación es significativa al nivel .01 (bilateral).

* La correlación es significante al nivel .05 (bilateral). 
Tabla 3

Medias, desviaciones estándar y pruebas de hipótesis para los efectos de redes atencionales sobre la propensión a cometer errores durante la conducción

\begin{tabular}{|c|c|c|c|}
\hline & AAPIDOS & HFTIM & $F[1, h)$ \\
\hline TH & $29+4$ (6. $\mathrm{kb})$ & $3326(10506)$ & 1123 \\
\hline ALIRTA & Mom (1004y) & $9121(7448)$ & 171 \\
\hline ATRNCION EJECTINA & $29.26(6.707)$ & $33.4406018)$ & 1.760" \\
\hline ORIENTACION & MI) $(\mathrm{R}, 453)$ & 31919754 & 200 \\
\hline
\end{tabular}

$*$ Significativo al nivel $\mathrm{p}<.05$

Tabla 4

Medias y pruebas de hipótesis para los efectos de interacción entre variables sociodemográficas y Tiempos de Reacción general sobre propensión al error

\begin{tabular}{|c|c|c|c|c|}
\hline & & ALTO TH & BANO TR & $\mathbf{P}[1, \mu]$ \\
\hline \multirow{2}{*}{ Fdul } & Edid 1 & $31.25(4.7 \mathrm{n})$ & 30.48 (7.068) & \multirow{2}{*}{2400} \\
\hline & Inde & $5106(13670)$ & $2 x(6,7 x)$ & \\
\hline \multirow{2}{*}{ Seso } & Famenims & $3494(12525)$ & $2957(7291)$ & \multirow{2}{*}{1.919} \\
\hline & Masulha & Mo.s (s.R14) & $2923(\mathrm{k} .747)$ & \\
\hline \multirow{2}{*}{ Foprticia } & Enptw & 35.58 (13.602) & $26.5(5210)$ & \multirow{2}{*}{$7,746=$} \\
\hline & Nosula & 3033 (4.R6) & $33.46(7.783)$ & \\
\hline
\end{tabular}

* Significativo al nivel $\mathrm{p}<.01$ 
Tabla 5

Medias, desviaciones estándar, y pruebas de hipótesis de los efectos de redes atencionales para cada dimensión de ARDES

\begin{tabular}{|c|c|c|c|c|c|c|c|}
\hline \multirow{4}{*}{$\begin{array}{l}\text { TR } \\
(\mathrm{n}=68)\end{array}$} & \multirow{4}{*}{$\begin{array}{l}\text { Lento } \\
\text { Rápido }\end{array}$} & \multicolumn{2}{|l|}{ Maniobra } & \multicolumn{2}{|l|}{ Navegaciớn } & \multicolumn{2}{|l|}{ Control } \\
\hline & & $\mathrm{M}(\mathrm{DS})$ & $\mathrm{F}$ & $\mathrm{M}(\mathrm{DS})$ & $\mathrm{F}$ & $\mathrm{M}(\mathrm{DS})$ & F \\
\hline & & $13.76(4.214)$ & \multirow{2}{*}{2.951} & 8.79 (3.409) & \multirow{2}{*}{1.318} & $8.85(3.791)$ & \multirow{2}{*}{2.351} \\
\hline & & $12.18(3.362)$ & & $7.94(2.674)$ & & $7.62(2.775)$ & \\
\hline \multirow{2}{*}{$\begin{array}{l}\text { A lerta } \\
(n-69)\end{array}$} & Alta & $12.74(4.300)$ & \multirow{2}{*}{.161} & $8.43(3.128)$ & \multirow{2}{*}{.068} & $7.83(3.593)$ & \multirow{2}{*}{.966} \\
\hline & Bajo & $13.12(3.391)$ & & $8.24(3.036)$ & & $8.62(3.045)$ & \\
\hline \multirow{2}{*}{$\begin{array}{l}\text { Orientación } \\
(\mathrm{n}-68)\end{array}$} & Alta & $13.12(3.975)$ & \multirow{2}{*}{.188} & $8.26(2.885)$ & \multirow{2}{*}{.038} & $8.29(3.648)$ & \multirow{2}{*}{.062} \\
\hline & Bajo & $12.71(3.865)$ & & $8.41(3.331)$ & & $8.09(3.137)$ & \\
\hline \multirow{2}{*}{$\begin{array}{l}\text { A tención } \\
\text { Ejecutiva } \\
(\mathrm{n}=68)\end{array}$} & Alta & $13.59(4.200)$ & \multirow[b]{2}{*}{1.754} & $8.68(3.666)$ & \multirow[b]{2}{*}{.684} & $9.32(3.846)$ & \multirow[b]{2}{*}{$7.889^{*}$} \\
\hline & Bajo & $12.35(3.454)$ & & $8.06(2.348)$ & & $7.15(2.376)$ & \\
\hline
\end{tabular}

* Significativo al nivel $\mathrm{p}<.01$

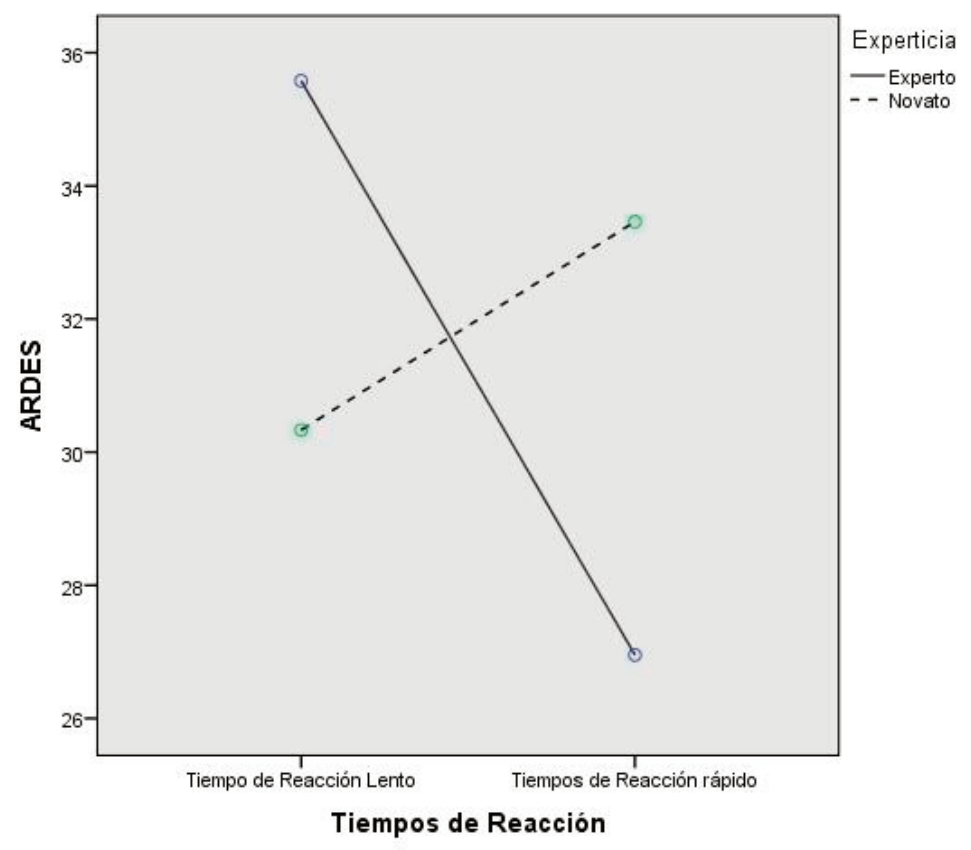

Figura 1. Interacción entre experticia y tiempos de reacción (atención general) sobre la propensión a cometer errores durante la conducción. 


\section{Referencias bibliográficas}

af Wåhlberg, A., \& Dorn, L. (2009). Bus driver accident record: the return of accident proneness. Theoretical Issues in Ergonomics Science, 10, 77-91.

Allahyari, T., Saraji, G.N., Adl, J., Hosseini, M., Iravani, M., Younesian, M., \& Kass, S. J. (2008). Cognitive failures, driving errors and driving accidents. International Journal of Occupational Safety \& Ergonomics, 14, 149-15. http://dx.doi.org/10.1080/108035 48.2008.11076759

Bradshaw, J.L., \& Nettleton, N.C. (1983). Human cerebral asymmetry. NJ: Prentice Hall.

Callejas, A., Lupianez, J., \& Tudela, P. (2004). The three attentional networks: On their independence and interaction. Brain and Cognition, 54, 225-227. http://dx.doi.org/ 10.1016/j.bandc.2004.02.012

Castro, C., Crundall, D., Chapman, P., Trawley, S., \& Underwood, G. (2009). Un-experienced vs. Experienced drivers. Limitations of Human Attention. An analysis of their THREE ATTENTIONAL NETWORKS. Paper presented to First International Conference on Driver Distraction and Inattention. Ontario, Canadá. Retrieved Septiembre 12, 2015, from https://document.chalmers.se/download?docid=130371 5557

Castro, M., Durán, D., \& Cantón, D. (2006). La conducción vista por los psicólogos cognitivos. Boletín de Psicología, 87, 35-60.

Centro de Experimentación y Seguridad Vial. (n.d.). Retrieved Agosto 20, 2015, from https://www.cesvi.com.ar/home.html

Clay, O.J., Wadley, V.G., Edwards, J.D., Roth, D.L., Roenker, D.L., \& Ball, K.K. (2005). Cumulative meta-analysis of the relationship between useful field of view and driving performance in older adults: current and fu- ture implications. Optometry and Vision Science: Official Publication of the American Academy of Optometry, 82(8), 724-731. http://dx.doi.org/10.1097/01.opx. 0000175009.08626 .65

Fan, J., \& Posner, M. (2004). Human attentional networks. Psychiatrische Praxis, $31(\mathrm{~S}$ 2), 210-214. http://dx.doi.org/10.1055/s2004-828484

Fan, J., McCandliss, B., Sommer, T., Raz, A., \& Posner, M. (2002). Testing the efficiency and independence of attentional networks. Journal of Cognitive Neuroscience (14), 340-347. http://dx.doi.org/10.1162/089892 902317361886

Groeger, J. (2002). Trafficking in cognition: applying cognitive psychology to driving. Transportation Research part F: Traffic Psychology and Behaviour, 5, 235-248. http://dx.doi.org/10.1016/S1369-8478(03) 00006-8

Kolb, B., \& Whishaw, I.Q. (1985). Fundamentals of human neuropsychology. New York: Freeman.

Larson, G.E., Alderton, D.L., Neideffer, M., \& Underhill, E. (1997). Further evidence on dimensionality and correlates of the Cognitive Failures Questionnaire. British Journal of Psychology, 88, 29-38. http://dx. doi.org/10.1111/j.2044-8295.1997.tb026 18. $\mathrm{x}$

Ledesma, R.D., Montes, S.A., Poó, F.M., \& López-Ramón, M.F. (2010). Individual Differences in Driver Inattention: The Attention -Related Driving Errors Scale. Traffic Injury Prevention, 11(2), 142-150. http://dx.doi. org/10.1080/15389580903497139

Ledesma, R., Montes, S., Poó, F., \& LópezRamón, M. (2015). Measuring Individual Differences In Driver Inattention. Further Validation of The Attention-Related Driving Errors Scale. Human Factors, 57(2), 193-207. http://dx.doi.org/10.1177/ 0018720814546530 
Lellis, V., Mariani, M., Ribeiro, A., Cantiere, C., Teixeira, M., \& Carreiro, L. (2013). Voluntary and automatic orienting of attention during childhood development. Psychology \& Neuroscience, 6(1), 15-21. https://dx.doi.org/10.3922/ j.psns.2013.1.04

López-Ramón, M.F., Castro, C., Roca, J., Ledesma, R., \& Lupiañez, J. (2011). Attentional Networks Functioning, Age, and Attentional Lapses While. Traffic Injury Prevention, 12, 518-528.

Lovett, M., Reder, L., \& Lebiere, C. (1999). Modeling Working Memory in a Unified Architecture: An ACT-R Perspective. En A. Miyake, \& P. Shah (Eds.), Models of Working Memory (pp. 135-182). Oxford University Press. http://dx.doi.org/10.1017/ CBO9781139174909.008

Luna, F.G., Marino, J., Macbeth, G., \& Torres, G.F. (2016). ¿Existen diferencias entre sexos en las redes atencionales? Una revisión sistemática sobre el alerta fásica, vigilancia, orientación y control ejecutivo. Psychologia, Avances de la disciplina, 10(2), 6371. Retrieved from http://www.redalyc.org/ articulo.oa? $\mathrm{id}=297248896006$

Michon, J. A. (1985). A critical view of driver behavior models: what do we know, what should we do? In Human behavior and traffic safety (pp. 485-524). Springer US. http://dx.doi.org/10.1007/978-1-4613-2173619

Miró, E., Martínez, M.P., Sánchez, A.I., Prados, G., \& Lupiáñez, J. (2014). Men and Women with Fibromyalgia: Relation Be-tween Attentional Function and Clinical Symptoms. British Journal of Health Psychology, 20(3), 632-647.

Mizobuchi, S., Chignell, M., \& Canell, D. (2013). Individual Differences in DrivingRelated Multitasking. 3rd International Conference on Driver Distraction and Inattention September 4-6, 2013, (pp. No. 72-P). Gothenburg, Sweden.
Montes, S. (2013). Diferencias individuales y correlatos psicológicos de los errores relacionados con la inatención en conductores. Mar del Plata, Buenos Aires, Argentina: Universidad Nacional de Mar del Plata.

Musso, M.F. (2016). Understanding the underpinnings of academic performance: The relationship of basic cognitive processes, self-regulation factors and learning strategies with task characteristics in the assessment and prediction of academic performance. Doctoral Dissertation, KU Leuven.

OMS. (2009). World Health Organization. Retrieved from WHO Library Cataloguingin-Publication Data: www.who.int/violence_injury_prevention/road_safety_ status/2009

Posner, M. (1980). Orienting of attention. Quarterly Journal of Experimental Psychology, 32, 23-25. http://dx.doi.org/ 10.1080/ 00335558008248231

Posner, M. (2008). Measuring alertness. Annals of the New York Academy of Sciences, 1129(1), 193-199. http://dx.doi.org/10.1196/ annals. 1417.011.

Posner, M. I. (1994). Attention: The mechanism of consciousness. Proceedings of the National Academy of Sciences of the United States of America, 91(16), 7398-7402. http://dx.doi.org/10.1073/pnas.91.16.7398

Posner, M. I., \& Petersen, S. E. (1990). The attention system of the human brain. Annual Review Neuroscience, 13, 25-42. http://dx. doi.org/10.1146/annurev.ne.13.030190.000325

Posner, M., \& Rothbart, M. (2007). Research on attention networks as a model for the integration of psychological science. Annual Review of Psychology, 58, 1-23. http://dx. doi.org/10.1146/annurev.psych.58.110405.0 85516

Reason, J. (1990). Human Error. New York: Cambridge University Press. http://dx.doi. org/10.1017/CBO9781139062367 
Roca, J., Lupiáñez, J., López- Ramón, M.F., \& Castro, C. (2013). Are drivers' attentional lapses associated with the functioning of the neurocognitive attentional networks and with cognitive failure in everyday life? Transportation Research Part F, 98-113.

Vaquero, E., Cardoso, M.J., Vazquez, M., \& Gomez, C.M. (2004). Gender differences in Event related potentials during visual-spatial attention. International of. Journal of Neuroscience, 114, 541-557. http://dx.doi. org/10.1080/00207450490422056

Victor, T., Engström, J., \& Harbluk, J.L. (2008). 10 Distraction Assessment Methods Based on Visual Behavior and Event Detection. Driver distraction: theory, effects, and mitigation, 135. http://dx.doi.org/10.1201/97814 20007497.ch10

Visser, E., Pijl, Y., Stolk, R., Neeleman, J., \& Rosmalen, J. (2007). Accident proneness, does it exist? A review and meta-analysis. Accident Analysis and Prevention, 39, 556564. http://dx.doi.org/10.1016/j.aap.2006. 09.012
Wallace, J.C., \& Vodanovich, S.J. (2003). Can accidents and industrial mishaps be predicted? Further investigation into the relationship between cognitive failure and reports of accidents. Journal of Business and Psychology, 17, 503-514.

Wang, H., \& Fan, J. (2007). Human Attentional Networks: A Connectionist Model. Journal of Cognitive Neuroscience, 19(10), 16781689. http://dx.doi.org/10.1162/jocn.2007. 19.10.1678

Weaver, B., Bédard, M., \& McAuliffe, J. (2013). Evaluation of a 10-minute Version of the Attention Network Test. The Clinical Neuropsychologist, 27(8), 1281-1299. http:// dx.doi.org/10.1080/13854046.2013.851741

Weaver, B., Bédarda, M., McAuliffe, J., \& Parkkari, M. (2009). Using the Attention Network Test to predict driving test scores. Accident Analysis and Prevention, 41, 7683. http://dx.doi.org/10.1016/j.aap.2008.09. 006

Recibido: 10 de diciembre de 2016 Aceptado: 10 de julio de 2018 